\title{
Repulsive Forces of Highly Coercive Permanent Magnets in Magnetic Clutches and Plane Magnetic Systems
}

\author{
A. Ya. Krasil'nikov ${ }^{a}$ and A. A. Krasil'nikov ${ }^{b}$ \\ ${ }^{a}$ Yeltsin Ural Federal University, Yekaterinburg \\ e-mail: krasilnikov1951@yandex.ru \\ ${ }^{b}$ OAO SverdNIIkhimmash, Yekaterinburg
}

\begin{abstract}
A formula is derived for calculating the repulsive force of highly coercive permanent magnets made from rare-earth metal alloys in frontal magnetic clutches and plane magnetic systems, when the magnets take the form of rectangular prisms.
\end{abstract}

DOI: $10.3103 / \mathrm{S} 1068798 X 13040114$

At present, frontal magnetic clutches and plane magnetic systems are widely used to ensure complete sealing of the interior of equipment (pumps, compressors, mixers, transporters, and hoists) and to protect operating personnel and the environment from toxic materials [1]. The main deficiency of frontal magnetic clutches and plane magnetic systems is the presence of attractive and repulsive forces between the opposing permanent magnets in their components. These forces increase with decrease in the air gap between the magnets.

In normal operating conditions of sealed equipment, attractive forces act between the half-clutches of the frontal magnetic clutches (along the axis of rotation) or the sections in the plane magnetic systems (perpendicular to their direction of motion) [2]. Those forces act on the roller or slip bearings of drive shafts and driven shafts in machines or sealed transporters.

In emergencies, when the torque on the executive organ or the tractional force is greater than the limiting value, the magnetic coupling between the components is lost. If matching of the half-clutches or magnetic sections is disrupted, then those components will be attracted together (with consistent magnetization of the magnets) or repelled (with opposing magnetization of the magnets). Loss of matching results in alternating loads.

It follows from the foregoing that, at the design stage, the attractive and repulsive forces of permanent magnets must be determined. The calculation of the attractive forces was considered in [2].

We assume that the attractive and repulsive forces of the permanent magnets in the system components are equal and opposite. We will verify this hypothesis experimentally.

The experiments are conducted on a special test bench. In Fig. 1, we show the configuration of the sections in plane magnetic system with highly coercive permanent magnets when measuring the repulsive

forces. In Figs. 2 and 3, we present the experimental dependence of the unit attractive $\left(F_{\text {un.at }}\right)$ and repulsive $\left(F_{\text {un.re }}\right)$ forces in the sections of the plane magnetic system on the air gap $\delta$ between the magnets. The table presents the values of the unit attractive and repulsive forces.

The unit repulsive force of the magnets is calculated from the formula

$$
F_{\text {un.re }}=F_{\text {re }} / S \text {, }
$$

where $F_{\text {re }}$ is the repulsive force of the magnets in the plane magnetic system, N; $S$ is the surface area (upper or lower) of the magnets, $\mathrm{cm}^{2}$.

Analysis shows that the unit repulsive forces for most magnets are less than the unit attractive forces. The unit forces are approximately equal for the air gap $\delta=12 \mathrm{~mm}$. The same is true for magnets that are not rectangular prisms (Fig. 4).

(a)
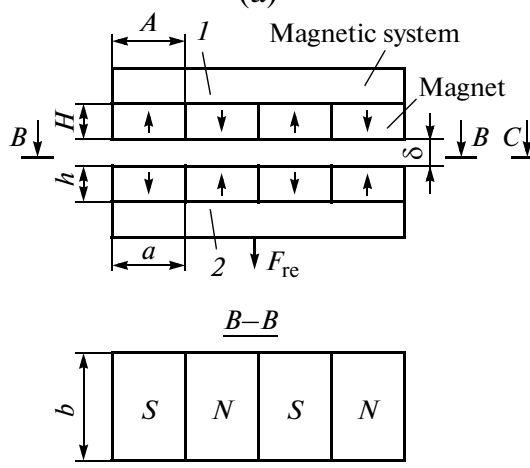

Fig. 1. Configuration of the sections in a plane magnetic system when measuring the repulsive force $F_{\text {re }}$, for magnets in the form of rectangular and trapezoidal prisms; (1) upper section; (2) lower section; ( $\uparrow$ ) direction of magnetization. 
(a)

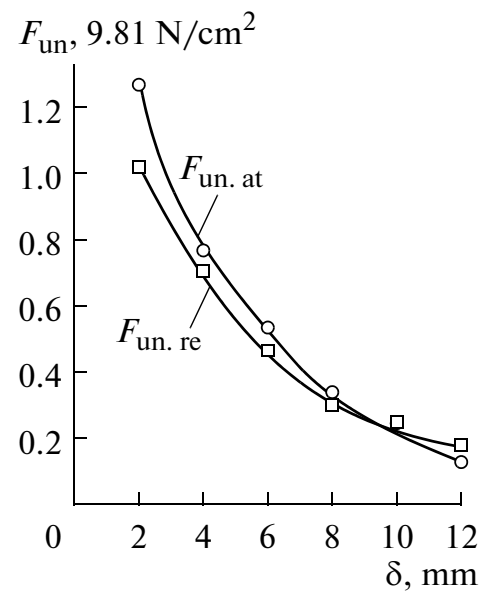

(b)

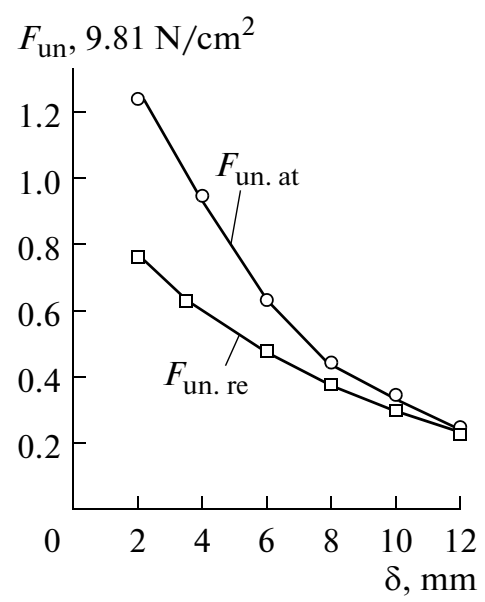

Fig. 2. Experimental dependence of the unit attractive $\left(F_{\text {un.at }}\right)$ and repulsive $\left(F_{\text {un.re }}\right)$ forces on the air gap $\delta$ in the case of $15 \times 40 \times 8 \mathrm{~mm}$ (a) and $20 \times 30 \times 10 \mathrm{~mm}$ (b) permanent magnets made from group-1 KS-37 alloy $\left(B_{r}=0.76 \mathrm{~T}\right)$.

Frontal magnetic clutches and plane magnetic systems with $\delta=3-8 \mathrm{~mm}$ are widely used. As is evident from the table, the unit repulsive forces within this range are no more than $90 \%$ of the unit attractive forces. Therefore, the overall repulsive force between the components of the frontal magnetic clutch or plane magnetic system may be calculated from Eq. (11) of [2]

$$
\begin{gathered}
F_{\mathrm{re}}=0.9 F \\
=0.9\left[1.33\left(B_{r n \mathrm{lim}} / B_{r 1 \mathrm{lim}}\right)^{2}-0.57(\delta / \Delta)\right] m F_{z} .
\end{gathered}
$$

With air gaps $\delta>8 \mathrm{~mm}$, we recommend that the total repulsive force between the components be assumed equal to the total attractive force

$$
F_{\mathrm{re}}=F=\left[1.33\left(B_{r n \lim } / B_{r 1 \lim }\right)^{2}-0.57(\delta / \Delta)\right] m F_{z} .
$$

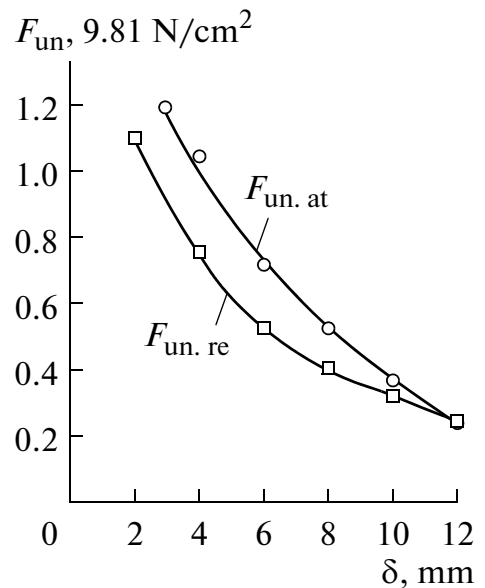

Fig. 3. Experimental dependence of the unit attractive $\left(F_{\text {un.at }}\right)$ and repulsive $\left(F_{\text {un.re }}\right)$ forces on the air gap $\delta$ in the case of $18 \times 40 \times 8 \mathrm{~mm}$ permanent magnets made from group-2 KS-37 alloy $\left(B_{r}=0.80 \mathrm{~T}\right)$.

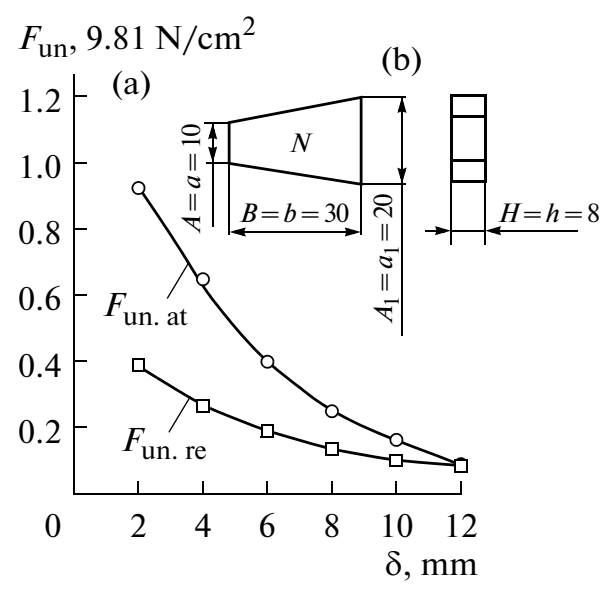

Fig. 4. Experimental dependence (a) of $F_{\text {un.at }}$ and $F_{\text {un.re }}$ on the air gap $\delta$ in the case of $10(20) \times 30 \times 8 \mathrm{~mm}$ permanent magnets made from group- $1 \mathrm{KS}-37$ alloy $\left(B_{r}=0.76 \mathrm{~T}\right)$ and trapezoidal configuration of magnets (b).

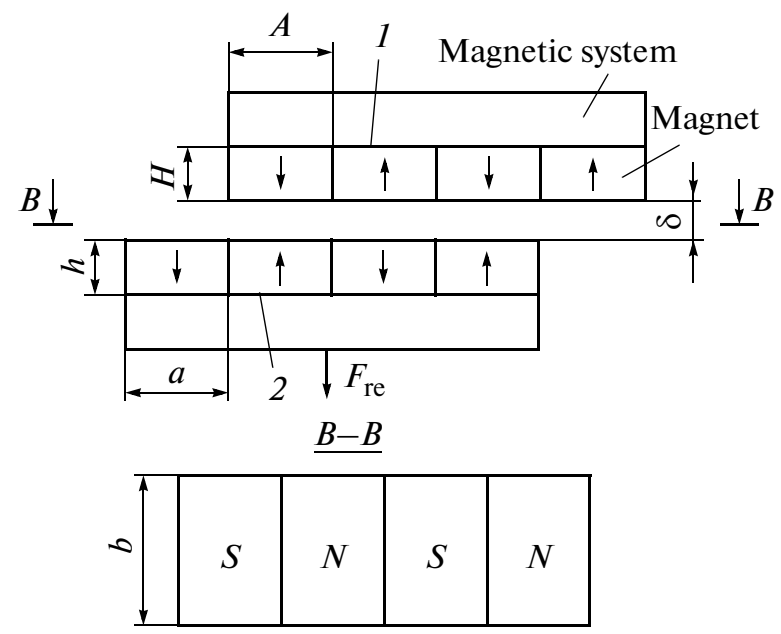

Fig. 5. Configuration of upper (1) and lower (2) sections of a plane magnetic system with repulsive forces: $(\uparrow)$ direction of magnetization. 
Experimental values of unit attractive $\left(F_{\text {un.at }}\right)$ and repulsive $\left(F_{\text {un.re }}\right)$ forces

\begin{tabular}{|c|c|c|c|c|}
\hline $\begin{array}{c}\text { KS-37 magnets } \\
\text { (group; dimensions, mm) }\end{array}$ & $\delta, \mathrm{m}$ & $F_{\text {un.at }}, \mathrm{N} / \mathrm{cm}^{2}$ & $F_{\text {un.re }}, \mathrm{N} / \mathrm{cm}^{2}$ & $F_{\text {un.re }} / F_{\text {un.at }}$ \\
\hline \multirow{6}{*}{$\begin{array}{l}\text { Rectangular prismatic mag- } \\
\text { nets (group } 1 ; 15 \times 40 \times 8 \text { ) }\end{array}$} & 2 & 12.65 & $10.1(2.2)$ & $0.84(2.2)$ \\
\hline & 4 & 7.75 & 6.97 & 0.90 \\
\hline & 6 & 5.40 & 4.41 & 0.87 \\
\hline & 8 & 3.34 & 2.94 & 0.88 \\
\hline & 10 & 2.16 & 2.45 & 1.13 \\
\hline & 12 & 1.375 & 1.86 & 1.35 \\
\hline \multirow{6}{*}{$\begin{array}{l}\text { Rectangular prismatic mag- } \\
\text { nets (group } 1 ; 20 \times 30 \times 10 \text { ) }\end{array}$} & 2.3 & 12.36 & $7.55(2.0)$ & 0.60 \\
\hline & 4.0 & 9.52 & $6.08(3.5)$ & 0.62 \\
\hline & 6.0 & 6.38 & 4.61 & 0.72 \\
\hline & 8.0 & 4.41 & 3.68 & 0.83 \\
\hline & 10.0 & 3.43 & 2.94 & 0.86 \\
\hline & 12.0 & 2.45 & 2.26 & 0.92 \\
\hline \multirow{6}{*}{$\begin{array}{l}\text { Rectangular prismatic mag- } \\
\text { nets (group } 2 ; 18 \times 40 \times 8 \text { ) }\end{array}$} & 3 & 11.97 & $10.89(2.0)$ & 0.76 \\
\hline & 4 & 10.30 & 7.41 & 0.72 \\
\hline & 6 & 7.23 & 5.16 & 0.71 \\
\hline & 8 & 5.30 & 4.02 & 0.76 \\
\hline & 10 & 3.73 & 3.14 & 0.86 \\
\hline & 12 & 2.35 & 2.45 & 1.04 \\
\hline \multirow{6}{*}{$\begin{array}{l}\text { Trapezoidal prismatic mag- } \\
\text { nets }(\text { group } 1 ; 0(20) \times 30 \times 8) \\
{\left[A=a_{1}=10 ; A_{1}=a_{1}=20\right]}\end{array}$} & 2 & 9.22 & 3.92 & 0.43 \\
\hline & 4 & 6.47 & 2.65 & 0.41 \\
\hline & 6 & 3.921 & 1.86 & 0.47 \\
\hline & 8 & 2.45 & 1.28 & 0.52 \\
\hline & 10 & 1.67 & 0.98 & 0.59 \\
\hline & 12 & 0.83 & 0.73 & 0.88 \\
\hline
\end{tabular}

Note: Values of $\delta$ different from those in the given column are given in parentheses.

In practice, with mismatch (rupture) of the magnetic coupling, one section of the magnetic system remains motionless, while the other moves horizontally by a single magnet, as shown in Fig. 3 of [3]. In the other words, the contact includes $(m-1)$ pairs of magnets, where $m$ is the number of magnets in a single component of the system (Fig. 5). In that case, Eqs. (1) and (2) take the form

$$
\begin{gathered}
F_{\mathrm{re}}=0.9 F \\
=\left[1.33\left(B_{r n \lim } / B_{r 1 \mathrm{lim}}\right)^{2}-0.57(\delta / \Delta)\right](m-1) F_{z} ; \\
F_{\mathrm{re}}=F \\
=\left[1.33\left(B_{r n \mathrm{lim}} / B_{r 1 \mathrm{lim}}\right)^{2}-0.57(\delta / \Delta)\right](m-1) F_{z} .
\end{gathered}
$$

The results permit calculation of the total repulsive force of the highly coercive permanent magnets in the system. That force may be taken into account in the design as the additional force (load) in selecting the roller bearings.
Our research also shows that, when $\delta=3-8 \mathrm{~mm}$, the repulsive force is less than the attractive force between the half-clutches in the frontal magnetic clutch. In calculating the repulsive force within that range, a value of $90 \%$ of the attractive force may be assumed. When $\delta>$ $8 \mathrm{~mm}$, the attractive and repulsive forces are close (when $\delta=12 \mathrm{~mm}$, they are practically equal), and they may be assumed equal in the calculations.

\section{REFERENCES}

1. Krasil'nikov, A.Ya., Standard Designs of Magnetic Systems and Clutches, Khim. Neftegaz. Mashinostr., 2002, no. 7, pp. 35-37.

2. Krasil'nikov, A.Ya. and Krasil'nikov, A.A., Calculating the Attractive Forces of Highly Coercive Permanent Magnets in Frontal Magnetic Clutches and Plane Magnetic Systems, Vestn. Mashinostr., 2010, no. 6, pp. 13-16.

3. Krasil'nikov, A.Ya. and Krasil'nikov, A.A., Calculating the Interaction Forces of Highly Coercive Permanent Magnets as a Function of Their Group, Vestn. Mashinostr., 2009, no. 9, pp. 17-19. 